\title{
DROPLET ARRAYS IN MICROFLUIDIC CHANNELS FOR COMBINATORIAL SCREENING ASSAYS
}

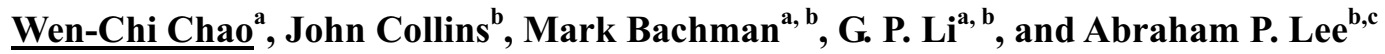

${ }^{\mathrm{a}}$ Department of Electrical and Computer Engineering, ${ }^{\mathrm{b}}$ Department of Biomedical Engineering, and

${ }^{c}$ Department of Mechanical \& Aerospace Engineering,

University of California at Irvine

A microfluidic droplet array that allows dynamic reconfiguration of droplet volume and concentration is reported. Microfluidic droplet technology [1, 2] shows tremendous promise for high throughput combinatorial screening assays by varying the chemical compositions in successive droplets [3]. However, in order to study long term (hours to days) chemical changes (e.g. protein crystallization, enzymatic reactions) inside droplets, active trapping is necessary for temporal and spatial analysis of each droplet in place. The major challenge is to avoid droplet coalescence that would destroy the encapsulated content. Stabilization methods include polymerization and the addition of surfactants to the droplets, but these may ultimately affect the evaporation rate and the chemical content of the droplet [4]. In addition, the ability to successively vary the sizes of the droplets is critical for nucleation and solubility assays based on the evaporation rate of the droplet $[5,6]$.

The droplet array microfluidic chip is shown in Fig 1, which utilizes a PDMS channel junction to generate droplet emulsions as described in [1]. For trapping droplets, a

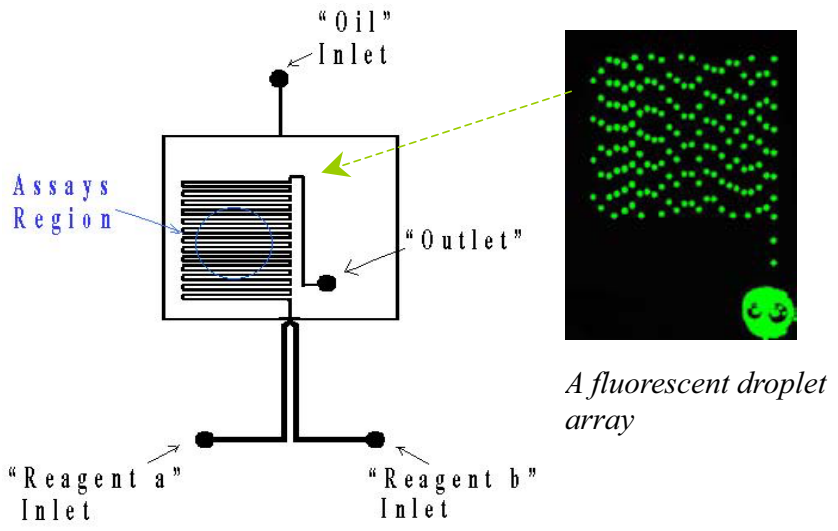

Fig. 1. Schematics of microfluidic chip.

zero-dead-volume single-pole double-flow valve from the Lee Company served to divert the flow from the inlets. The control pulses applied to these valves are in phase with each other so that the valves are synchronized. However, many manufacturers claim their microfluidic component to have "zero-dead-volume" when in reality there exits a small dead volume. In addition, even after the flow is switched to a shunt outlet, residual pressure will continue to generate uncontrolled droplets; therefore, trapping droplet arrays in place is challenging. In order to overcome these two problems (dead volume and residual pressure), after the Lee valve diverts the inlet flow to the shunt, the inlet is subsequently exposed to atmospheric pressure in a "timely" manner. The purpose is to control the volume of flow "released" towards the outlet to be balanced by the volume "returned" to the inlet. By doing this process, the droplet arrays are trapped in place to be identified as shown in Fig 2.

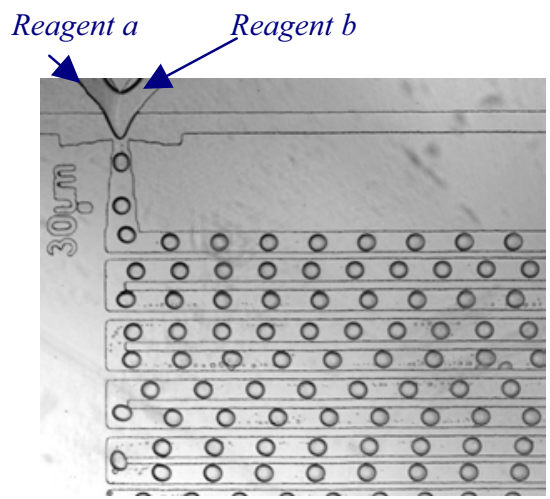

Fig. $2 a$. A droplet array carrying two reagents in flow

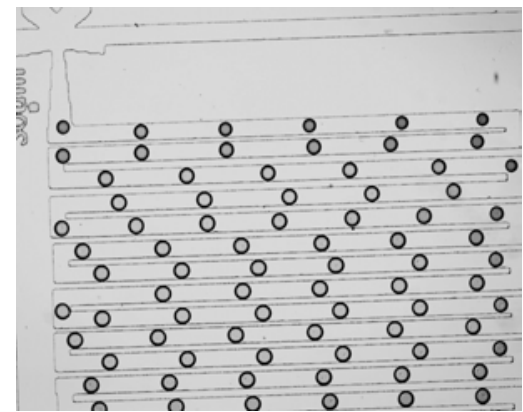

Fig. 2b. A droplet array trapped in microfluidic channels for screening. After trapping, the droplet is round due to the disappearance of the viscous shearing forces.

In our experiments, the droplet sizes and chemical concentrations are ramped by programming the flow rates of oil and one of the chemical reagents, respectively, by using Harvard 2000 syringe pumps, controlled by an RS232

Travel support has been generously provided by the Transducers Research Foundation and by the DARPA MEMS and DARPA BioFlips programs. 
interface. Preliminary results are shown in Fig 3 and Fig 4 with droplets having volume gradients and chemical gradients, respectively. As these plots illustrate the sizes of the droplets were more easily controlled (Fig 3) than the concentration (Fig 4). In Fig 3 and Fig 4, $Q_{a}$ is the flow rate of reagent $a ; Q_{b}$ is the flow rate of reagent $b$, and $Q_{o}$ is the flow rate of mineral oil. As seen in Fig. 4, the end pinching effect results in instability of concentration ramping. The images are recorded with a high-speed camera at 500 frames per second.
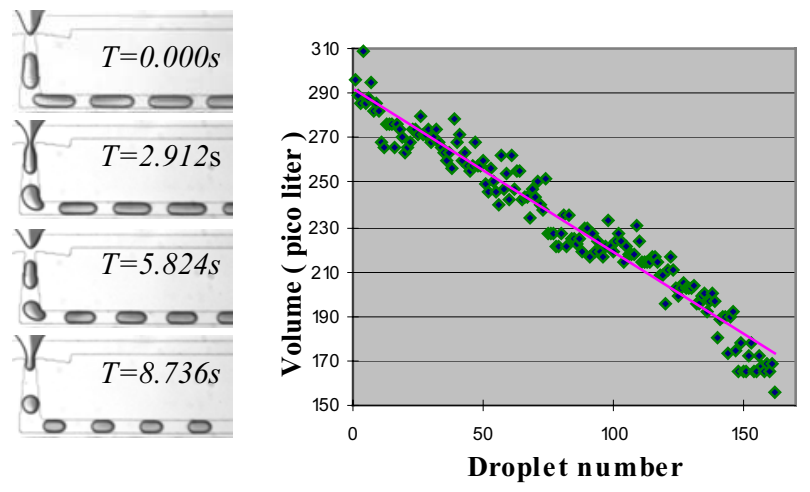

Fig. 3. Dynamic droplet volume ramped in 8.736 s shown in a series of video frames. $Q_{a}=0.7 \mu \mathrm{l} / \mathrm{min}, Q_{b}=1.3 \mu \mathrm{l} / \mathrm{min}$, and $Q_{o}$ $=0.4 \sim 2.4 \mu \mathrm{l} / \mathrm{min}$
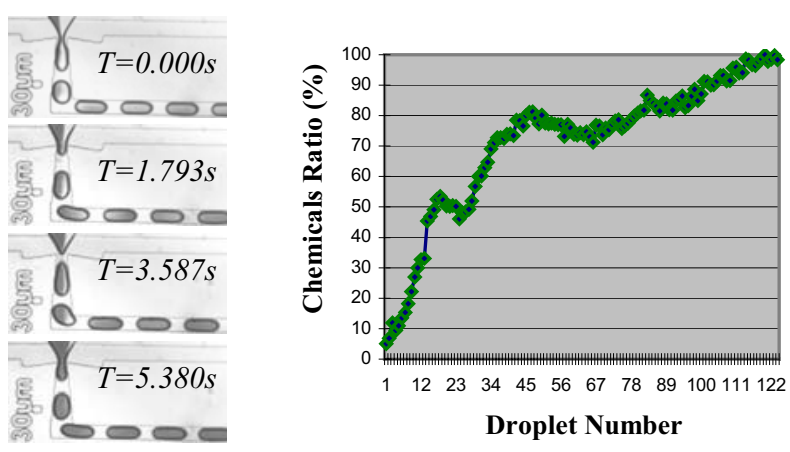

Fig. 4. Dynamic chemical concentration ramped in $5.380 \mathrm{~s}$ shown in a series of video frames. $Q a=0.7 \mu l / \mathrm{min}, Q b$ $=0.6 \sim 3.0 \mu \mathrm{l} / \mathrm{min}$, and $Q o=1.3 \mu \mathrm{l} / \mathrm{min}$.

A preliminary experiment of a combinatorial screening assay is demonstrated by observing salt solubility in droplets containing $\mathrm{NaCl}$ solution. As shown in Fig 5, crystallization occurred in droplets having higher salt concentration; however, temperature is an important parameter in salt crystallization as shown in Fig 6, as the solubility is higher at higher temperatures. Our droplet device forms a platform to generate multidimensional solubility diagrams for various chemicals.

\section{REFERENCES}

[1] Yung-Chieh Tan, John Collins, and Abraham P. Lee, 7th

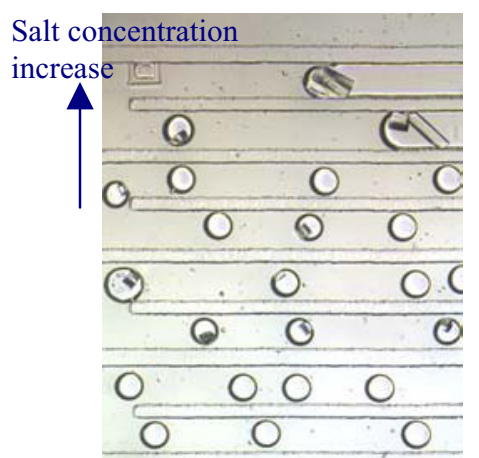

Fig. 5. Solubility assay based on the salt concentration

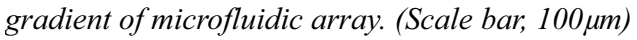

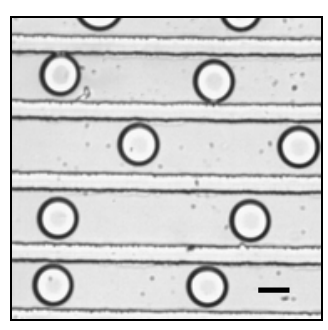

Temp. $=25^{\circ} \mathrm{C}$ right after trapping

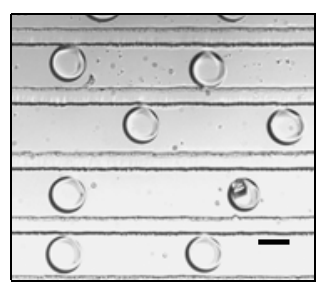

Temp. $=5^{\circ} \mathrm{C}$ fort 20 mins

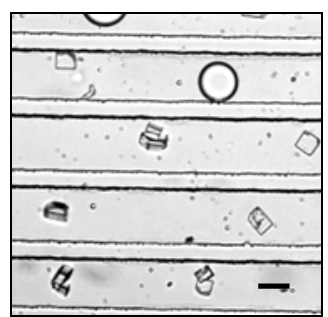

Temp. $=-10^{\circ} \mathrm{C}$ for $1 \mathrm{hrs}$

Fig. 6. Salt crystallization observed as the temperature cooled down. (Scale bar, 50 $\mathrm{m}$ )

International Conference on Miniaturized Chemical and Biochemical Analysts Systems, October 5-9, 2003

[2] Helen Song, Joshua D. Tice, and Rustem F. Ismagilov,

Angew. Chem. Int. Ed. 2003, 42, No.7

[3] Michael Spaid, Andrea Chow, Yevgeny Yurkovetsky,

Micro Total Analysis Systems 2003, 445-448, October 2003

[4] T. Thorsen, R.W. Roberts, F.H. Arnold and S.R. Quake, Phys. Rev. Lett. 86: 4163-6 (2001)

[5] P. D. Collingsworth, Terry L. Bray, Gayle K. Christopher, Crystal Growth 219, 283-289 (2000).

[6] Bo Zheng, L. Spencer Roach, and Rustem F. Ismagilov, J. American Chemical Society 2003, 125, 11170-11171 